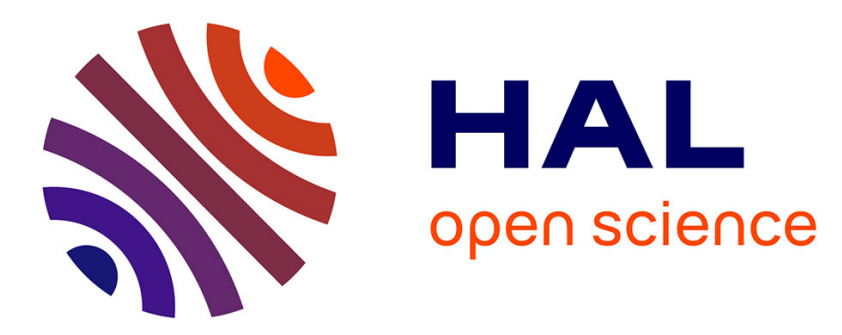

\title{
Noise characterization of depth sensors for surface inspections
}

François Pomerleau, Andreas Breitenmoser, Ming Liu, Francis Colas, Roland Siegwart

\section{- To cite this version:}

François Pomerleau, Andreas Breitenmoser, Ming Liu, Francis Colas, Roland Siegwart. Noise characterization of depth sensors for surface inspections. 2nd International Conference on Applied Robotics for the Power Industry (CARPI), 2012, Zurich, Switzerland. 10.1109/CARPI.2012.6473358 . hal01142707

\section{HAL Id: hal-01142707 https://hal.science/hal-01142707}

Submitted on 15 Apr 2015

HAL is a multi-disciplinary open access archive for the deposit and dissemination of scientific research documents, whether they are published or not. The documents may come from teaching and research institutions in France or abroad, or from public or private research centers.
L'archive ouverte pluridisciplinaire HAL, est destinée au dépôt et à la diffusion de documents scientifiques de niveau recherche, publiés ou non, émanant des établissements d'enseignement et de recherche français ou étrangers, des laboratoires publics ou privés. 


\title{
Noise Characterization of Depth Sensors for Surface Inspections
}

\author{
François Pomerleau, Andreas Breitenmoser, Ming Liu, Francis Colas, Roland Siegwart
}

\begin{abstract}
In the context of environment reconstruction for inspection, it is important to handle sensor noise properly to avoid distorted representations. A short survey of available sensors is realize to help their selection based on the payload capability of a robot. We then propose uncertainty models based on empirical results for three models of laser rangefinders: Hokuyo URG04LX, UTM-30LX and the Sick LMS-151. The methodology, used to characterize those sensors, targets more specifically different metallic materials which often give distorted images due to reflexion. We also evaluate the impact of sensor noise on surface normal vector reconstruction and conclude with observations about the impact of sunlight and reflexions.
\end{abstract}

\section{INTRODUCTION}

In many inspection applications, a 3D model of the environment needs to be constructed either as a final product or as a support to other information like thickness, heat, etc. The Magnebike is an example of a mobile robotic platform capable of inspecting power plant facilities with focus on the inner casing of steam chests [1]. This mobile system has demonstrated the feasibility of environment reconstruction in a confined space. Figure 1 shows an example of such reconstruction using the rotating laser scanner Hokuyo URG04LX. Although the reconstruction is globally consistent, one can observe systematic noisy readings in the lower part of the 3D model. Those deformations can impact the quality of $3 \mathrm{D}$ registration algorithms. Noise characterization for light and portable laser rangefinders would lead to more robust 3D mapping algorithms and better define their applicability to structural inspection in industrial environments.

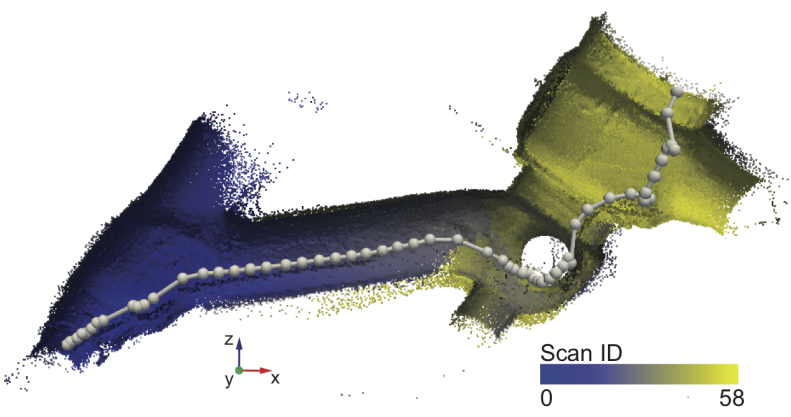

Fig. 1. Reconstruction of a rusty metallic steam chest. The color represents incremental scan ID through time. The gray line represents the path of the robot.

Other researchers have already evaluated and characterized various depth sensors. Therefore, we present in the first section a survey of those results, highlighting limitations and common challenges. We also present a list of depth sensors that are actually available on the market and we categorize them into different types of mobile systems. The second section describes our results from 3 laser rangefinders, namely the Hokuyo URG-04LX, UTM-30LX, and the Sick LMS-151, tested in different conditions. We conclude by proposing specific error models aiming at reducing the impact of faulty measurements on registration algorithms like the Iterative Closest Point (ICP).

\section{RELATED WORK}

In the literature, rangefinder sensor characterizations evaluate depth measurement errors under the variation of one or multiple conditions. A first group of characterizations evaluates the depth reading with respect to the target distance, incidence angle, brightness/reflectivity and heat level (radiance). Further studies evaluate the robustness of measurement to different ambient lights and air conditions (e.g., dust, rain, smoke, etc.). Finally, range sensors were found to be sensitive to their internal temperature which can evolve in time, specially during the first hour of utilization. This type of error can easily be in the range of centimeters [2]. The mixed pixel problem (i.e., a single laser beam footprint can be large enough to cover 2 objects at different depth) was first highlighted in [3] and is still present in more recent sensors.

The most studied scanner is the Sick LMS-200, which was first characterized in [4] and then further investigated in [5], [6], [7], [8], and [9]. A new generation of smaller Sick laser (LMS-100 family) was evaluated in [10]. This new scanner was presented as a competition to the smaller scanners proposed by Hokuyo. Evaluations for the Hokuyo series was well investigated with the characterization of the PBS-03JN [5], the UBG-04LX-F01 [11], the URG-04LX [7], [2], [12], [13] and, more recently, the UTM-30LX [14], [15], [16]. Comparisons between Sick LMS-200 and Hokuyo URG scanners were also tackled [8], [7]. At a larger scale, Wong et al. [17] compare 10 sensors, from the Faro Photon 80 up to a custom made stereo ring, in an underground mapping situation. Time-of-flight cameras, from Mesa Imaging (formally SwissRanger), were characterized in [18] and [19], which leaded to calibration models. Calibration for the Velodyne was also proposed in [20] to improve the accuracy of the scanner from $23 \mathrm{~cm}$ to $1.5 \mathrm{~cm}$. Recently, the Kinect was used in many robotics applications and was also characterized in [21]. Those studies, for sensors that are not discontinued, are regrouped in Table I to ease further consultation.

Many more rangefinder sensors exist on the market and their selection for an inspection task can depend on different 
TABLE I

RELATED STUDIES OF POPULAR RANGE SENSORS IN RELATION WITH DIFFERENT TYPES OF EVALUATIONS

\begin{tabular}{|c|c|c|c|c|c|c|}
\hline & Kinect & $P B S$ & $U B G$ & $U R G$ & UTM & $\begin{array}{l}L M S \\
200\end{array}$ \\
\hline \multicolumn{7}{|l|}{ Target } \\
\hline distance & [21] & {$[5]$} & [11] & $\begin{array}{l}{[13][2]} \\
{[7]}\end{array}$ & {$[15]$} & [7] [4] \\
\hline incidence angle & & {$[5]$} & & $\begin{array}{l}{[13][2]} \\
{[7]}\end{array}$ & & [7] [4] \\
\hline brightness & [22] & {$[5]$} & $\begin{array}{l}{[22]} \\
{[11]}\end{array}$ & $\begin{array}{l}{[13][2]} \\
{[7]}\end{array}$ & & [7] [4] \\
\hline reflectivity & & {$[5]$} & [11] & $\begin{array}{l}{[13][2]} \\
{[7]}\end{array}$ & & [7] [4] \\
\hline heat & & & & [7] & & [7] \\
\hline \multicolumn{7}{|l|}{ Ambient } \\
\hline $\begin{array}{l}\text { lights } \\
\text { conditions }\end{array}$ & [14] & & & $\begin{array}{l}{[2]} \\
{[7]}\end{array}$ & [15] [14] & [7] \\
\hline \multicolumn{7}{|l|}{ Other } \\
\hline $\begin{array}{l}\text { sensor attitude } \\
\text { mixed pixel } \\
\text { time/temp. drift }\end{array}$ & & $\begin{array}{l}{[5]} \\
{[5]}\end{array}$ & [11] & $\begin{array}{l}{[2]} \\
{[13]} \\
{[13][2]} \\
{[8][7]}\end{array}$ & {$[15]$} & $\begin{array}{l}{[9][4]} \\
{[6][8} \\
{[7][4]}\end{array}$ \\
\hline
\end{tabular}

criteria. For example, when external power is not accessible, power consumption of the sensors becomes critical. Also, a large maximum range of the sensor may become essential if an environment is difficult to access, or covers a large area. Field of view (FOV) can also impact the design of mobile inspection tools since too narrow FOV will require extra actuation to offer a better coverage of the environment. We propose in Fig. 2 an overview of the market by representing 25 sensors with respect to their maximal range versus their weight. Payload that can be carried by a mobile platform may be a limiting factor of small systems. This figure is intended to help in defining a first group of sensors that a platform could carry while quickly seeing the possible scanning range accessible to the platform. On the yaxis, we can also divide the graph into airborne survey sensors with more than $1 \mathrm{~km}$ range, terrestrial survey sensors around $100 \mathrm{~m}$ to $1 \mathrm{~km}$, and safety/robotics sensors under $100 \mathrm{~m}$.

Finally, new technologies are emerging in this growing field. More specifically at the level of early products and development kits, it is worth noting the TigerEye 3D Flash LIDAR, proposed by Advanced Scientific Concepts, which can produce a depth image with a single laser beam. The system can read from a distance of up to $70 \mathrm{~m}$ with a 45 degrees lens. Another company, Lytro, proposes a small and low cost camera producing depth images with a single lens. They use a device called light field to capture simultaneously multiple focus points and reconstruct a depth field out of the image. Hand-held 3D scanner with sub-millimeter precision also exist for reverse engineering or culture heritage preservation as offered by Artec 3D or Creaform, to name a few. This type of sensor offers more precision at the expense of range.

Based on Fig. 2, we selected sensors with weight small enough to be carried in an inspected environment while ensuring a reasonable scanning range. We focused on time-

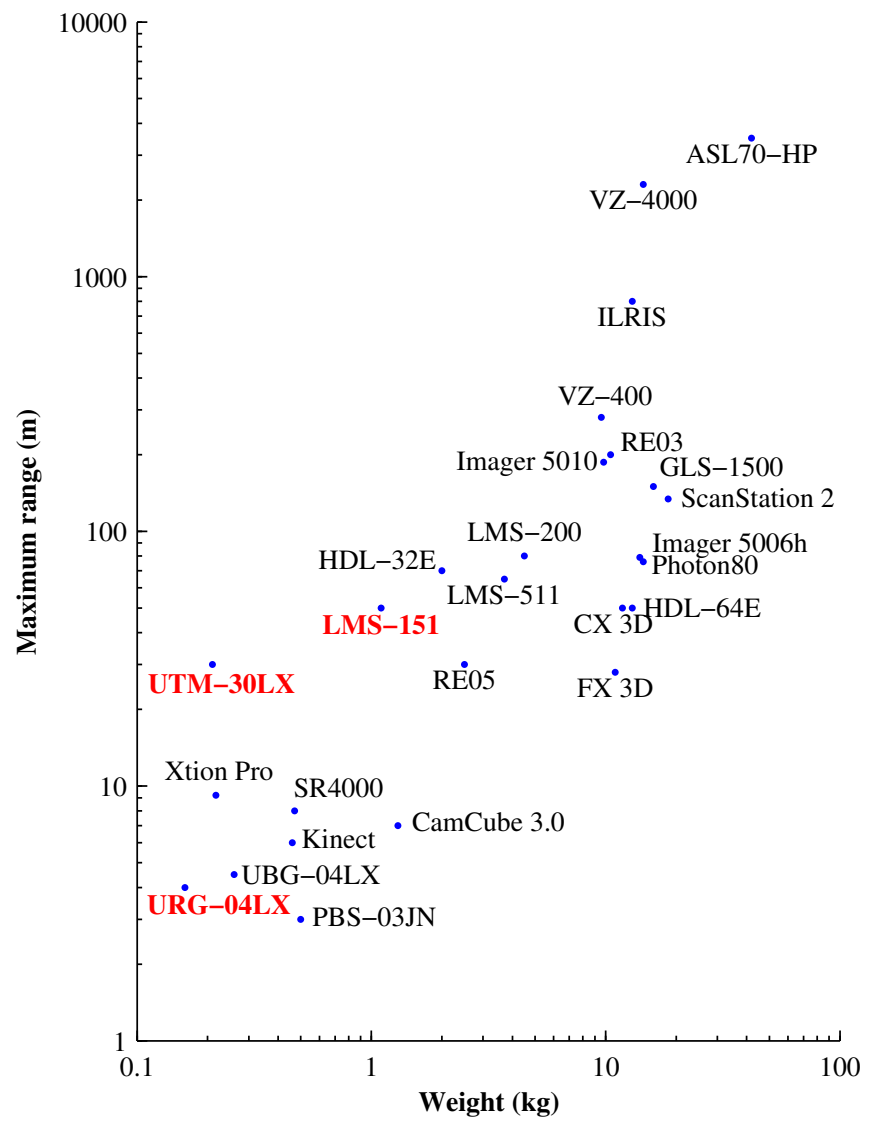

Fig. 2. Classification of range sensors based on maximum range (m) and weight $(\mathrm{kg})$. Note $\log$ scale. The sensors evaluated in this paper are in bold red. Manufacturers: Mesa Imaging (SR4000), PMD (CamCube 3.0), ASUS (Xtion Pro), Microsoft (Kinect), Occular Robotics (RE05, RE03), Hokuyo (UBG-04LX, URG-04LX, UTM-30LX), Sick (LMS-151, LMS-200, LMS511), Velodyne (HDL-64E, HDL-32E), FARO (Photon80), Leica (ScanStation 2, ASL70-HP), Riegl (VZ-400, VZ-4000), Topcon (GLS-1500), Trimble (FX 3D, CX 3D), Optec (ILRIS), and Zoller+Froehlich (Imager 5010, Imager $5006 \mathrm{~h})$.

of-flight and phase-shift depth sensors to ensure uniformity in the evaluation methodology. We have investigated the UTM30LX and the LMS-151 to provide uncertainty models for which no models were found in the literature. Finally, we have also evaluated the URG-04LX with the same methodology to compare our results with prior characterizations [2], [13], and [7].

\section{EXPERIMENTAL PROTOCOL}

As opposed to most of the protocols used in formal characterization, which focused on a single beam, we realized our evaluation on full 3D scans of metallic plates. We believe that this type of evaluation is much closer to real applications and leads to more realistic models. We used the custom made tilting platform, described in more details in [23], to mount the 3 different lasers and take 3D scans of different surfaces. The surfaces have been selected to represent a subset of metallic surfaces that could be found in the power industry installations while covering a large spectrum of reflectance. The surfaces 
measured roughly $2 \mathrm{~m}$ long per $1 \mathrm{~m}$ large and the materials were: aluminum, metallic surface covered with paint (white board usually used with colored markers), old steel and rusty iron. Fig. 3 presents photographs of all plates.

We scanned those surfaces at 5 different distances, namely $0.2,1.0,2.0,4.0$ and $8.0 \mathrm{~m}$. The theodolite TS15, from Leica Geosystem, was used to collect ground truth distances in a precision range of millimeters. The poses (i.e., orientations and positions) of the scanner were determined with the same protocol as in [23]. As for the plate poses, 6 markers were installed on the periphery of the plates and measured with the same theodolite. Given that we have the transformation from the theodolite to the scanner, we can also express the points on the plates in the reference frame of the scanner. Using the location of those markers, the plate is divided in 4 triangles against which all points from the 3D scan of the plate are segmented (Fig. 4 - top). With some basic geometry concepts, the error of each beam was determined given the triangle in which it is associated (Fig. 4 - middle). For each 3D scan, the intensity reflected by the surface was also recorded (Fig. 4 - bottom). Those measurements were realized indoors for all combinations of sensors, materials and distances. The experimental setup was repeated outdoors for the the UTM$30 \mathrm{LX}$ to observe the influence of the sun on the measurements. The illumination of the environment at the plate level was measured with the TES 1332a Digital LUX Meter. This gave us a total of 80 different 3D scans with an average of 30,000 points per scan.

Moreover, another set of measurements has been done to determine the width of the laser beams. Each sensor scanning plane was put perpendicular to a wall at 5 different distances: $1,3,5$ and $9 \mathrm{~m}$. For each distance, a picture was taken using a camera without an infrared filter. A ruler fixed on the wall was used to determine the beam diameter as shown in Fig. 5.

\section{RESUlts}

\section{A. Random noise}

For all results reported in this subsection on noise modeling, we removed measurements that were heavily affected by reflection. In our data set, those points were producing errors larger than $0.05 \mathrm{~m}$. We separated the evaluation of those spurious readings and addressed them in Section IV-D.

We first analyzed the angular uncertainty for each laser. We used a linear regression in the form of $y=a x+b$ to evaluate the beam opening angle. For all fittings, the coefficient of determination $R^{2}$ was larger than 0.95 . Based on those results, we could confirm the manufacturer specifications for the LMS-151 with an opening angle of $0.83 \mathrm{deg}$. Hokuyo does not provide such specifications for the URG-04LX and the UTM-30LX. Based on our measurements, we evaluated the angles to be respectively $0.13 \mathrm{deg}$ and $0.14 \mathrm{deg}$.

For the uncertainty on depth, we used the measurement realized over different distances and clustered them per sensor and material as depicted in Fig. 6. For every type of material, represented in the figure with letters, the median is marked

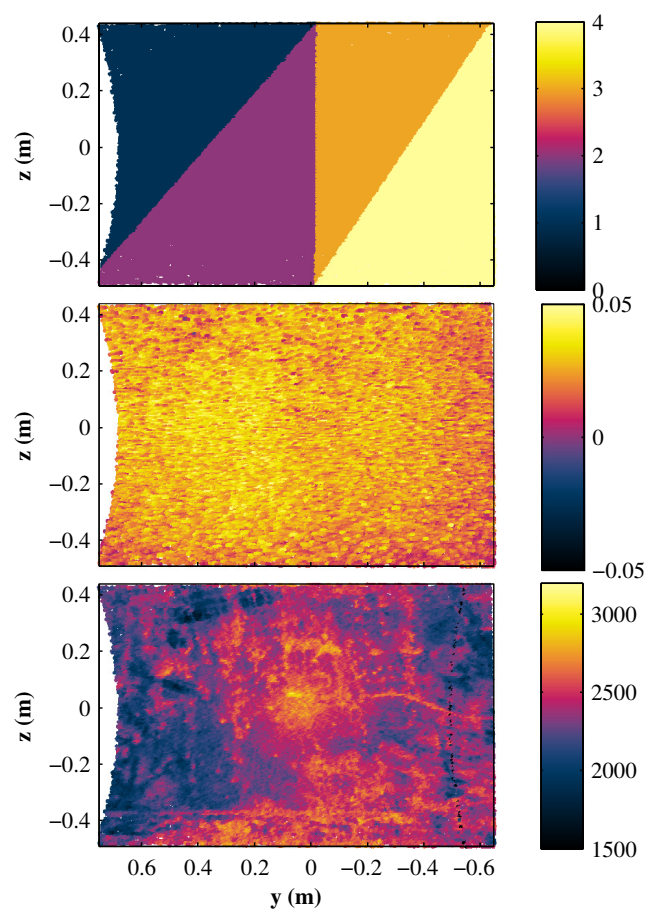

Fig. 4. Example of the results based on a scan of the iron plate at $1.0 \mathrm{~m}$ using the UTM-30LX. Top: point segmentation based on ground truth measurements, color representing the clusters. Middle: depth error on each beam value (positive when too close and negative when too far from the plane), color represents error in meter. Bottom: intensity returned for each beam, color represents the intensity as outputted from the UTM.

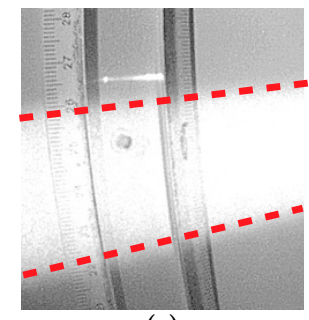

(a)

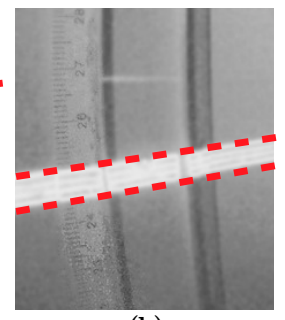

(b)

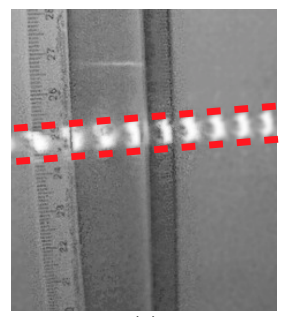

(c)
Fig. 5. Different laser patterns at $3 \mathrm{~m}$ : (a) LMS-151, (b) UTM-30LX and (c) URG-04LX.

with a point while the $68 \%$ of the measurements are contained within the error bars. Although systematic noise can be observed, especially for the URG-04LX given different materials, we were mainly interested in the disparity of the depth measurement error for the model. Considering that all types of materials are equally likely to be found in the environment, we used the mean of the distances between each error bar as an estimate of the standard deviation (stddev). This leads to a disparity of $0.028 \mathrm{~m}$ for the URG-04LX, $0.018 \mathrm{~m}$ for the UTM-30LX, and $0.012 \mathrm{~m}$ for the LMS-151.

Combining the angular and depth uncertainty, we defined 2 types of error models for every point based on the depth returned by the sensors. Both models approximate the dispersion 


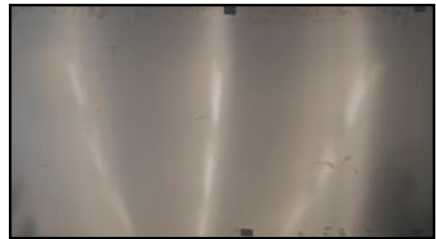

(a)

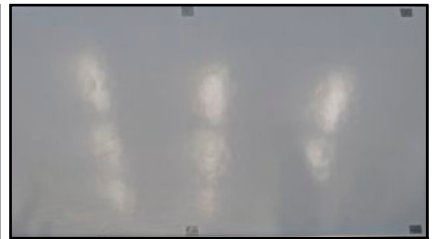

(b)

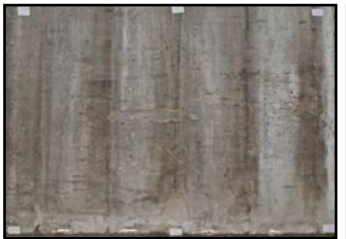

(c)

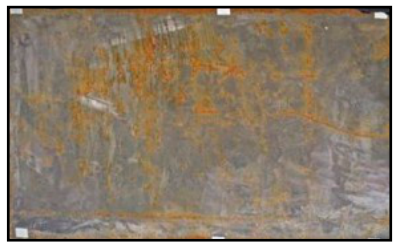

(d)

Fig. 3. Plates used for the experiments: (a) Aluminum, (b) White board, (c) Steel and (d) Rusted iron.

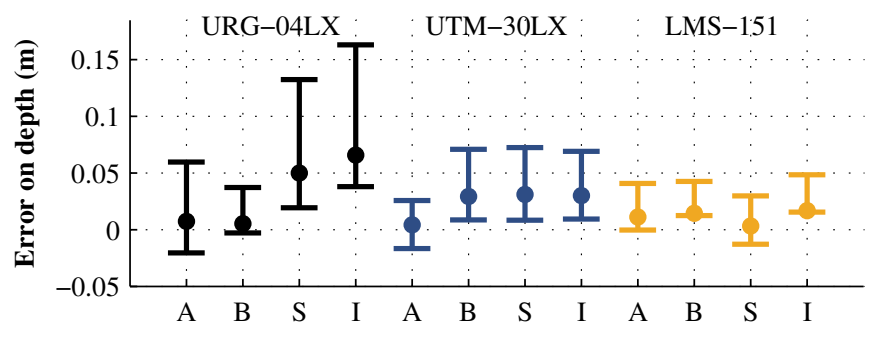

Fig. 6. Error in depth measurement for different types of material (A: Aluminum, B: White board, S: Steel, and I: Iron).

using Gaussian representation with the mean being the point read. The first model is anisotropic and is parametrized with a vector representing the beam direction $\vec{b}$ supporting the stddev on depth $\sigma_{d}$. The stddev of the beam radius $\sigma_{r}$ is supported implicitly by any vector perpendicular to $\vec{b}$. The second model is a further simplification using an isotropic representation with only one stddev defined as $\sigma_{m}=\max \left(\sigma_{d}, \sigma_{r}\right)$. Fig. 7 presents the notation and the comparison between the two models.

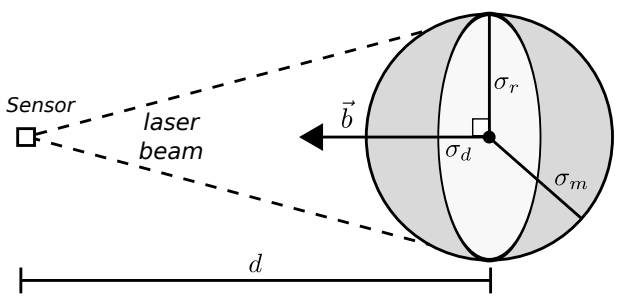

Fig. 7. 2D projection of the isotropic (gray) and the anisotropic (light gray) error model. In $3 \mathrm{D}, \sigma_{m}$ corresponds to the radius of a ball while $\sigma_{r}$ is uniform around $\vec{b}$

Parameters estimated for all sensors, based on our characterization, are resumed in Table II. A graphical comparison of the isotropic noise model for the 3 laser ranger finders is depicted in Fig. 8. We added the Kinect noise model from Khoshelham et al. [21] for comparison. One should be careful when comparing the LMS-151 and the Kinect because the largest uncertainty of the Kinect is mainly on depth measurements while the LMS-151 uncertainty is mainly caused by the laser beam opening angle. We can also observe that more precise measurements can be obtained with the Kinect if the expected structure is within $4 \mathrm{~m}$. Otherwise, the UTM-30LX should be used. On one side, the opening angle of the laser used for the LMS-151 produces less precise measurements at long range. On the other side, this opening angle coupled with its angular resolution ensures an overlap of $50 \%$ of every beam by the subsequent one, giving more safety insurance about the reading. The LMS-151 also has a longer range than the UTM-30LX.

TABLE II

UNCERTAINTY MODEL PARAMETERS FOR THE 3 LASERS STUDIED. THE MEASURED DEPTH $d$ IS EXPRESSED IN METERS.

\begin{tabular}{|c|c|c|c|}
\hline Sensor & Anisotropic & Isotropic & \\
\hline \multirow[t]{2}{*}{$U R G-04 L X$} & $\sigma_{r}=\frac{1.3 d-0.1}{1000}$ & $\sigma_{m}=0.028$ & \\
\hline & $\sigma_{d}=0.028$ & & \\
\hline \multirow[t]{2}{*}{$U T M-30 L X$} & $\sigma_{r}=\frac{0.6 d+1.48}{1000}$ & $\sigma_{m}=0.018$ & \\
\hline & $\sigma_{d}=0.018$ & & \\
\hline \multirow[t]{2}{*}{$L M S-151$} & $\sigma_{r}=\frac{6.8 d+0.81}{1000}$ & $\sigma_{m}=0.012$ & if $d<1.646$ \\
\hline & $\sigma_{d}=0.012$ & $\sigma_{m}=\frac{6.8 d+0.81}{1000}$ & else \\
\hline
\end{tabular}

When comparing our model for the URG-04LX to other studies, 2 main differences appear. First, we didn't correct the depth measurements based on a linear [13] or third order polynomial [2] correction. Based on our measurements, we couldn't find a simple model that was only based on the measured depth that could be useful in most inspected environments. Second, our stddev is roughly ten times larger than the ones suggested in the other studies. We argue that formal stddev reported are under estimated mainly due to the methodology used. The other evaluations take into consideration a single beam on an optimal material (medium gray [13] or white paper [2]), without considering the impact of the incidence angle. When looking more carefully at a larger range of error they reported over different incidence angles and materials, one can easily observe variance in the order of centimeters, which seems to be consistent with our model.

\section{B. Sensitivity to structure extraction}

In many ICP algorithms, structural information plays a critical role in the minimization process. A typical way to extract surface normal vectors from a point is to search for its nearest neighbors (NN), then recenter this subset to their mean and finally select the Eigen vector associated to the 

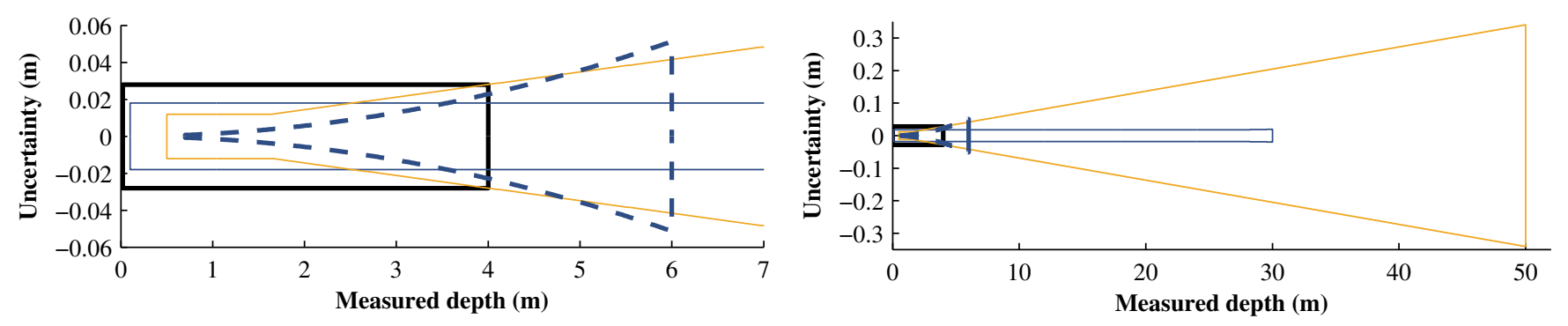

URG-04LX - UTM-30LX- LMS-151- - Kinect

Fig. 8. Comparison of the isotropic error model for the 3 lasers studied compared with the error model of the Kinect from [21].

smallest Eigen value as the surface normal. On one side, the maximal radius for which the NN search is bounded depends on the size of the expected planar structure. The larger this radius is, the more robust to noise the extracted planar surface will be. On the other side, the lower bound for the NN search radius depends of the expected noise of the sensor used. We characterized this sensitivity to noise by extracting surface normal for each point (all sensors, all distances and all materials) and variated the size of the NN search radius, $r=\{0.01,0.05,0.1,0.2,0.5\} \mathrm{m}$.

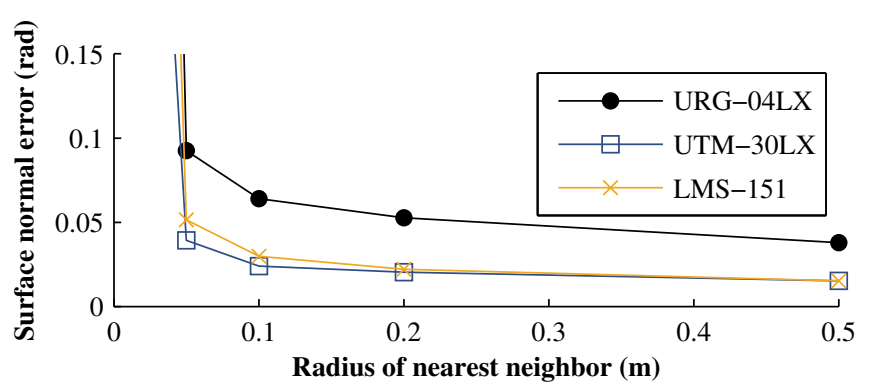

Fig. 9. Surface normal errors (rad) for different NN search radius for the 3 sensors studied.

Fig. 9 presents the median of the resulting surface normal error (rad) for each sensor over the tested radius. Given that the evaluation was done on large planar surfaces, one can observe that the error slowly decreases with a larger radius. Also, the error grows very quickly under $0.05 \mathrm{~m}$ for all sensors. Based on those observations, we propose this value as the minimum radius that should be used for surface extraction. Within that lower bound, we can expect to have surface normal error around $3.5 \mathrm{deg}(0.06 \mathrm{rad})$ for the URG-04LX and around $1.6 \mathrm{deg}(0.03 \mathrm{rad})$ for the UTM-30LX and the LMS-151. Of course, those values will hold only in the case where the density of point is high enough so the noise can be minimized.

\section{Impact of sunlight}

We could observe a difference between the measurements taken indoors and outdoors. The link between the sunlight and the difference in the reading is not that obvious. Prior evaluations concluded that internal temperature of the sensor can influence greatly the reading (see Table I column: time/temp. drift for references). The main influence might come from the sun heating the sensors. More controlled experiments must be conducted to confirm this hypothesis.

\section{Impact of reflection and large intensity range}

Reflecting surfaces like the aluminum plate poses 3 challenges. First, when the incidence angle is large, most of the energy is not reflected back to the sensor, which can lead to missing measurements. Based on Fig. 10 a), the URG04LX seems to be more sensitive to this phenomena than the others. Second, there is also the probability that the beam gets reflected to another surface leading to an overestimation of the depth. On Fig. $10 \mathrm{c}$ ), the lower part of the plate displayed a larger error due to the ground being reflected. We can conclude that the LMS-151 is more prone to such reflection given that the energy emitted by the sensor must be stronger to reach $50 \mathrm{~m}$. Finally, reflective plates exhibit a larger spectrum of reflected intensity, which seems to create systematic error producing wave patterns. Preliminary results show that there is a strong correlation between the error and the intensity.

\section{CONCLUSION}

In this paper, we proposed 2 types of model for the random noise of the URG-04LX, the UTM-30LX and the LMS-151 scanning laser rangefinders. We believe that the methodology used here provides more realistic models than in prior studies. Evaluation of surface reconstruction was also tackled, leading to the conclusion that a NN search radius should be larger than $5 \mathrm{~cm}$ to overcome the noise of the sensors. We intent to implement those models in libpointmatcher, a generic point cloud registration library [24]. Further studies will focus on the correlation between intensity and the depth error leading to systematic error patterns on reflective surfaces.

\section{ACKNOWLEDGMENT}

The research presented here was supported by the EU FP7 IP projects Natural Human-Robot Cooperation in Dynamic Environments (ICT-247870), and ALSTOM. F. Pomerleau was supported by a fellowship from the Fonds québécois de recherche sur la nature et les technologies (FQRNT). 
a)
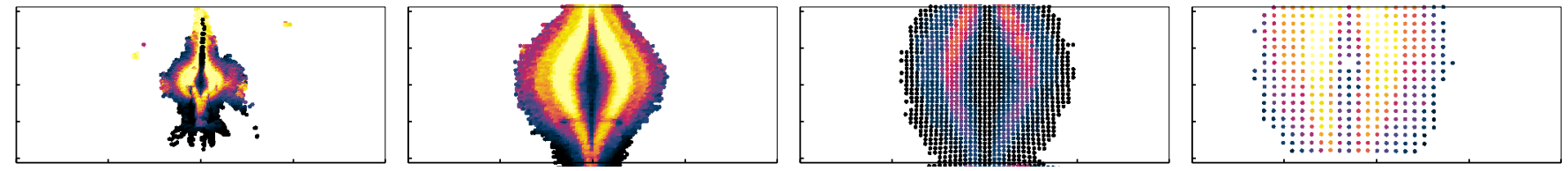

b)
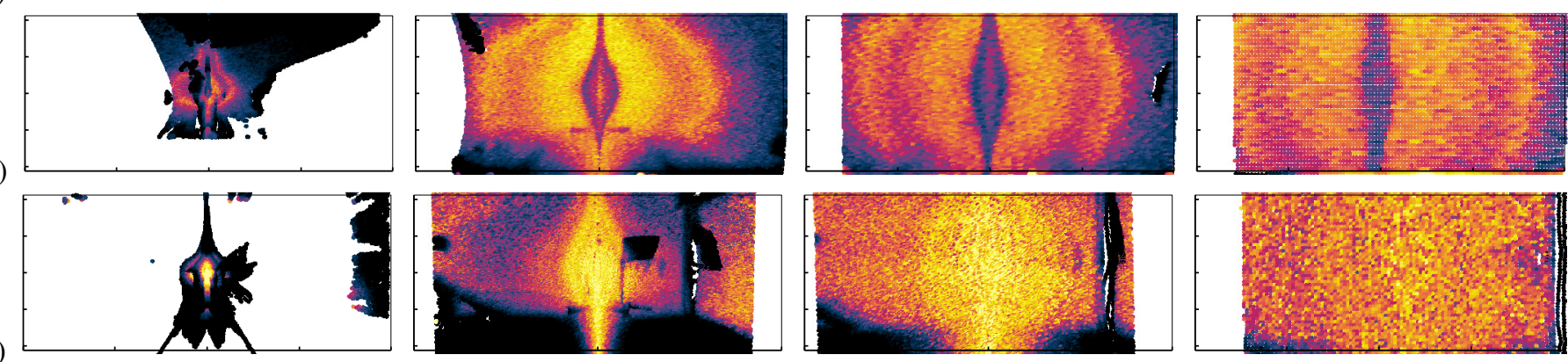

$-0.05$ $-0.03$ $-0.02$ $-0.01$ 0 0.01 0.02 0.03 $0.04 \quad 0.05$

Fig. 10. Front view of the aluminum plate for different distances (from left to right: 0.2, 1.0, 2.0, 4.0 m) and for a) URG-04LX, b) UTM-30LX, and c) LMS-151. Color represents the error on depth with black being the error mainly due to reflection.

\section{REFERENCES}

[1] F. Tâche, F. Pomerleau, G. Caprari, R. Siegwart, M. Bosse, and R. Moser, "Three-Dimensional Localization for the MagneBike Inspection Robot," Journal of Field Robotics, vol. 28, no. 2, pp. 180-203, 2011.

[2] L. Kneip, F. Tâche, G. Caprari, and R. Siegwart, "Characterization of the compact Hokuyo URG-04LX 2D laser range scanner," Robotics and Automation, 2009. ICRA '09. IEEE International Conference on, pp. 1447-1454, 2009.

[3] M. Hebert and E. Krotkov, "3-D measurements from imaging laser radars: how good are they?" in Intelligent Robots and Systems '91. 'Intelligence for Mechanical Systems, Proceedings IROS '91. IEEE/RSJ International Workshop on, 1991, pp. 359-364.

[4] C. Ye and J. Borenstein, "Characterization of a 2D laser scanner for mobile robot obstacle negotiation," in Robotics and Automation, 2002. Proceedings. ICRA 'O2. IEEE International Conference on. Washington: IEEE, 2002, pp. 2512-2518.

[5] M. Alwan, M. Wagner, G. Wasson, and P. Sheth, "Characterization of Infrared Range-Finder PBS-03JN for 2-D Mapping," in Robotics and Automation, 2005. ICRA 2005. Proceedings of the 2005 IEEE International Conference on, 2005, pp. 3936-3941.

[6] S. Jain, S. Nandy, G. Chakraborty, C. Kumar, R. Ray, and S. Shome, "Error modeling of Laser Range Finder for robotic application using time domain technique," in Signal Processing, Communications and Computing (ICSPCC), 2011 IEEE International Conference on, 2011, pp. 1-5.

[7] J. Pascoal, L. Marques, and A. T. de Almeida, "Assessment of Laser Range Finders in risky environments," in 2008 IEEE/RSJ International Conference on Intelligent Robots and Systems (IROS 2008). Nice: IEEE, 2008, pp. 3533-3538.

[8] K.-H. Lee and R. Ehsani, "Comparison of two 2D laser scanners for sensing object distances, shapes, and surface patterns," Computers and Electronics in Agriculture, vol. 60, no. 2, pp. 250-262, Mar. 2008.

[9] R. Sanz-Cortiella, J. Llorens-Calveras, J. R. Rosell-Polo, E. GregorioLopez, and J. Palacin-Roca, "Characterisation of the LMS200 Laser Beam under the Influence of Blockage Surfaces. Influence on 3D Scanning of Tree Orchards," Sensors, vol. 11, no. 3, pp. 2751-2772, Mar. 2011.

[10] J. Rudan, Z. Tuza, and G. Szederkenyi, "Using LMS-100 laser rangefinder for indoor metric map building," Industrial Electronics (ISIE), 2010 IEEE International Symposium on, pp. 525-530, 2010.

[11] C.-S. Park, D. Kim, B.-J. You, and S.-R. Oh, "Characterization of the Hokuyo UBG-04LX-F01 2D laser rangefinder," RO-MAN, 2010 IEEE, pp. 385-390, 2010.

[12] G. Paul, D. Liu, N. Kirchner, and G. Dissanayake, "An effective exploration approach to simultaneous mapping and surface material- type identification of complex threedimensional environments," Journal of Field Robotics, vol. 26, no. 1112, pp. 915-933, 2009.

[13] Y. Okubo, C. Ye, and J. Borenstein, "Characterization of the Hokuyo URG-04LX laser rangefinder for mobile robot obstacle negotiation," Unmanned Systems Technology XI. Proceedings of the SPIE, 2009.

[14] V. Tretyakov and T. Linder, "Range sensors evaluation under smoky conditions for robotics applications," in 2011 IEEE International Symposium on Safety, Security, and Rescue Robotics (SSRR). Kyoto: IEEE, 2011, pp. 215-220.

[15] X. Li, G. M. Xiong, Y. W. Hu, W. B. Li, Y. Jiang, J. W. Gong, and H. Y. Chen, "Comparison of Single-Layer and Multi-Layer Laser Scanners for Measuring Characteristic," Applied Mechanics and Materials, vol. 128129, pp. 548-552, Oct. 2011.

[16] S. Hrabar, "An evaluation of stereo and laser-based range sensing for rotorcraft unmanned aerial vehicle obstacle avoidance," Journal of Field Robotics, vol. 29, no. 2, pp. 215-239, Jan. 2012.

[17] U. Wong, A. Morris, C. Lea, J. Lee, C. Whittaker, B. Garney, and R. Whittaker, "Comparative evaluation of range sensing technologies for underground void modeling," in Intelligent Robots and Systems (IROS), 2011 IEEE/RSJ International Conference on, 2011, pp. 3816-3823.

[18] T. Kahlmann, F. Remondino, and H. Ingensand, "CALIBRATION FOR INCREASED ACCURACY OF THE RANGE IMAGING CAMERA SWISSRANGER," ISPRS Commission V Symposium Image Engineering and Vision Metrology, 2006.

[19] S. May, D. Droeschel, D. Holz, S. Fuchs, E. Malis, A. Nüchter, and J. Hertzberg, "Three-dimensional mapping with time-of-flight cameras," Journal of Field Robotics, vol. 26, no. 11-12, pp. 934-965, Nov. 2009.

[20] G. Atanacio-Jimenez, J.-J. Gonzalez-Barbosa, J. B. Hurtado-Ramos, F. J. Ornelas-Rodriguez, H. Jimenez-Hernandez, T. Garcia-Ramirez, and R. Gonzalez-Barbosa, "LIDAR Velodyne HDL-64E Calibration Using Pattern Planes," International Journal of Advanced Robotic Systems, vol. 8, no. 5, pp. 70-82, 2011.

[21] K. Khoshelham and S. O. Elberink, "Accuracy and Resolution of Kinect Depth Data for Indoor Mapping Applications," Sensors, vol. 12, no. 2, pp. 1437-1454, Feb. 2012.

[22] C.-S. Park, S.-W. Kim, D. Kim, and S.-R. Oh, "Comparison of plane extraction performance using laser scanner and Kinect," in Ubiquitous Robots and Ambient Intelligence (URAI), 2011 8th International Conference on, 2011, pp. 153-155.

[23] F. Pomerleau, F. Colas, M. Liu, and R. Siegwart, "Challenging Data Sets for Point Cloud Registration Algorithms," International Journal of Robotics Research, 2012, To be published.

[24] F. Pomerleau, S. Magnenat, F. Colas, M. Liu, and R. Siegwart, "Tracking a depth camera: Parameter exploration for fast ICP," in Intelligent Robots and Systems (IROS), 2011 IEEE/RSJ International Conference on, 2011, pp. 3824-3829. 